\title{
Role of Sorbitol Accumulation and Myo-inositol Depletion in Paranodal Swelling of Large Myelinated Nerve Fibers in the Insulin-deficient Spontaneously Diabetic Bio-breeding Rat
}

\author{
Reversal by Insulin Replacement, an Aldose Reductase Inhibitor, and Myo-inositol
}

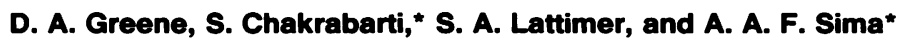 \\ Diabetes Research Laboratories of the Department of Medicine, University of Pittsburgh School of Medicine, Pittsburgh, Pennsylvania \\ 15160; *Neuropathology Research Laboratory, Department of Pathology, University of Manitoba, Winnipeg, Canada
}

\begin{abstract}
Axo-glial dysjunction refers to the disruption of important junctional complexes that anchor terminal loops of myelin to the paranodal axolemma in diabetic human and animal peripheral nerve. Neither axo-glial dysjunction nor the preceeding acute localized paranodal swelling has been specifically attributed to discrete metabolic consequences of insulin deficiency or hyperglycemia. Two metabolic sequelae of hyperglycemia in diabetic nerve, sorbitol accumulation via aldose reductase, and ( $\mathrm{Na}, \mathrm{K})$ ATPase deficiency related to myo-inositol depletion, were explored as possible underlying causes of acute paranodal swelling in the spontaneously diabetic bio-breeding rat. 3 wk of insulin replacement, or therapy with an aldose reductase inhibitor or myo-inositol completely reversed paranodal swelling in sural nerve fibers after 3 wk of untreated insulin deficiency. These observations suggest that insulin deficiency and hyperglycemia cause reversible paranodal swelling, and ultimately poorly reversible axo-glial dysjunction, via the myo-inositol-related (Na,K)-ATPase defect rather than by the osmotic effects of sorbitol accumulation within nerve fibers.
\end{abstract}

\section{Introduction}

In the last decade, a cascade of metabolic abnormalities in diabetic peripheral nerve, triggered by hyperglycemia and involving myo-inositol (MI) ${ }^{1}$ and $(\mathrm{Na}, \mathrm{K})-\mathrm{ATP}$ ase, has to a large extent satisfactorily explained the rapidly reversible slowing of nerve conduction in acutely diabetic rodents $(1,2)$; yet, the relationship of this metabolic cascade to the chronic and more pathogenetically relevant structural abnormalities characterizing both murine and human diabetic neuropathy remains to be clarified (2, 3 ). Activation of the polyol pathway by hyperglycemia appears to be an essential triggering event, since aldose reductase inhibitors (ARI) prevent MI depletion (4-6), the associated (Na,K)-

Address reprint requests to Dr. Greene, 5510C MSRB-I, 1150 West Medical Center Drive, University of Michigan Medical Center, Ann Arbor, MI 48109.

Presented in part at the Annual Meeting of the American Society for Clinical Investigation, Washington DC, May 1986.

Received for publication 18 September 1986.

1. Abbreviations used in this paper: $\mathrm{ARI}$, aldose reductase inhibitor; $\mathrm{BB}$, Bio-breeding; EMPA, evoked muscle potential amplitude; IDDM, insulin-dependent diabetes mellitus; MI, myo-inositol; MNCV, motor nerve conduction velocity; PZI, protamine zinc insulin.

J. Clin. Invest.

(c) The American Society for Clinical Investigation, Inc.

0021-9738/87/05/1479/07 \$1.00

Volume 79, May 1987, 1479-1485
ATPase defect (7), and the resultant reversible slowing of nerve conduction in diabetic animals (6); competitive inhibition by glucose of carrier-mediated MI uptake may also contribute to the depletion of nerve MI in diabetes (8). MI depletion diminishes nerve phosphoinositide turnover (9), interfering with $(\mathrm{Na}, \mathrm{K})$ ATPase regulation (9-13) by a protein kinase-C-dependent mechanism (14), thereby producing a four- to fivefold increase in intraaxonal sodium concentration $(1,3,15,16)$, inactivation of nodal sodium channels, and a selective conduction block of large myelinated nerve fibers that acutely slows composite saltatory conduction $(1,3,15,16)$. Increased intracellular sodium reduces the transmembrane gradient driving sodium-dependent MI uptake, further depleting MI and completing a self-reinforcing metabolic defect $(8,13)$.

The role of this metabolic and biophysical cascade in the development of the subsequent morphological abnormalities that characterize human and murine diabetic neuropathy has not been satisfactorily explored $(1,2)$. The initial characteristic morphometric abnormality in the peripheral neuropathy of the spontaneously diabetic Bio-breeding (BB) rat occurs within the first 3 wk of diabetes and consists of localized swelling of large myelinated nerve fibers in the region of the node of Ranvier (1, 3). This swelling and the accompanying four- to fivefold increase in intraaxonal sodium are prevented by insulin replacement therapy that normalizes blood glucose and nerve conduction velocity $(1,3)$. With more prolonged hyperglycemia, nodal swelling gives rise to axo-glial dysjunction (17) accompanied by apparent loss of functional sodium channels from the node, neither of which is acutely corrected by subsequent insulin replacement therapy despite rapid normalization of both MI content and $(\mathrm{Na}, \mathrm{K})-\mathrm{ATPase}$ activity $(13,17)$. Conceptually, the intracellular accumulation of either sorbitol (18) or sodium (3), through either aldose reductase activation or the MI-related (Na,K)-ATPase defect, respectively, could explain the early acute paranodal swelling thought to underlie axo-glial dysjunction. Hence, the ability of insulin replacement, ARI administration and MI supplementation to reverse paranodal swelling was explored in the acutely diabetic BB rat, whose fidelity as a model for human disease is highlighted by the recent identification of paranodal swelling and axo-glial dysjunction in sural nerve biopsies from patients with diabetic neuropathy (19). Insulin replacement, ARI administration, or MI supplementation completely reversed paranodal swelling as assessed by ratio of the paranodal fiber diameter to the internodal length. All three therapies correct $\mathrm{MI}$ depletion and $(\mathrm{Na}, \mathrm{K})-\mathrm{ATPase}$ inactivation, whereas only insulin replacement and ARI treatment but not MI supplementation prevent sorbitol accumulation $(6,20)$; hence, paranodal swelling in diabetic nerve most likely reflects the intracellular accumulation of sodium due to decreased $(\mathrm{Na}, \mathrm{K})-\mathrm{ATPase}$ activity rather than sorbitol accumulation. 


\section{Methods}

Experimental design and animal model. Prediabetic male BB-rats and age-matched nondiabetes-prone male BB-rats obtained from the Department of Pathology, University of Massachusetts, Worchester, MA (courtesy of Dr. A. Like), were maintained in individual air-filtered metabolic cages with ad lib. access to water and rat chow (Wayne Lab Blox F-6, Wayne Laboratory Animal Diets, Wayne Feed Division, Winnipeg, Manitoba [MI content $0.022 \% \mathrm{wt} / \mathrm{wt}$ ]) (17). Body weight, urine volume, and glucosuria (Testape, Eli Lilly Canada, Inc., Toronto, Ontario) were monitored daily, and glucose was measured in tail-vein blood samples by Ames Eyetone (Miles Laboratory, Ltd., Rexdale, Ontario) every second day between 2 and 4 p.m. (17). Upon detection of glucosuria and hyperglycemia, the newly diabetic rats were immediately begun on small daily doses $\left(0.5-3.0 \mathrm{U} \cdot \mathrm{d}^{-1}\right)$ of protamine zinc insulin (PZI), (Connaught Laboratories, Ltd., Toronto, Ontario), designed to maintain blood glucose levels between 350 and $450 \mathrm{mg} \cdot \mathrm{dl}^{-1}(17) .3 \mathrm{wk}$ thereafter, age-matched diabetic animals were randomly distributed into four experimental groups. "Baseline" diabetics were sacrificed immediately in order to obtain baseline structural and morphometric parameters (3). The other experimental groups were entered into a 3-wk study period during which: "insulin-deficient" diabetics were maintained hyperglycemic on small daily doses of PZI; "ARI-treated" diabetics were maintained insulin deficient on small doses of PZI but were given the ARI Statil (Stuart Pharmaceuticals, Wilmington, DE) $25 \mathrm{mg} \cdot \mathrm{kg}^{-1} \cdot \mathrm{d}^{-1}$ in a $10 \mathrm{mg} \cdot \mathrm{ml}^{-1}$ aqueous solution by gastric intubation; and "MI-treated" diabetics were maintained insulin deficient on small doses of PZI but in addition received MI, $670 \mathrm{mg} \cdot \mathrm{kg}^{-1} \cdot \mathrm{d}^{-1}$ in a $133-\mathrm{mg} \cdot \mathrm{ml}^{-1}$ aqueous solution by gastric intubation (6). These groups were compared to a similarly aged previously reported group of "insulin-replaced" diabetics treated aggressively with larger PZI doses $\left(3.0-6.0 \mathrm{U} \cdot \mathrm{d}^{-1}\right)$ adjusted for changes in blood glucose in order to achieve and maintain euglycemia (3). End-point measurements (nerve conduction, morphological and biochemical assessments) were performed by investigators unaware of the identity of the examined material.

Electrophysiological studies. Animals were lightly anesthetized with ethyl ether (Fisher Scientific Company, Fair Lawn, NJ). Motor nerve conduction velocity (MNCV) was determined noninvasively in the sciaticposterior tibial conduction system in a temperature controlled environment as previously described in detail (21). Briefly, the left sciatic-tibial nerve was stimulated proximally at the sciatic notch and distally at the ankle via bipolar electrodes using supramaximal stimuli $(8 \mathrm{~V})$ from a stimulater (TM 501, Tektronix, Inc., Beaverton, OR) at $20 \mathrm{~Hz}$. Evoked muscle potential amplitudes (EMPA) were collected from the first interosseous space of the hindpaw by a unipolar platinum recording electrode and displayed on a Tektronix 511 storage oscilloscope. Motor nerve conduction velocity was calculated by subtracting the distal from the proximal latency measured in milliseconds from stimulus artifact to take off of the evoked muscle potential; the resultant difference was divided into the distance between the stimulating electrodes measured in millimeters, yielding a value for MNCV in meters per second. MNCV and EMPA were measured on a weekly basis throughout the study (21).

Tissue collection. On the day after the final MNCV determination, nonfasted animals were anesthetized with Na pentobarbital $\left(50 \mathrm{mg} \cdot \mathrm{kg}^{-1}\right)$. Mid-thigh segments of the left and right sciatic nerves were surgically removed, weighed, and processed either for enzymatic ATPase measurements or for gas-liquid chromatographic determination of MI content as previously described in detail (11). The right proximal sural nerve (opposite to the side on which MNCV was performed) was fixed in situ for $10 \mathrm{~min}$ by a cacodylate buffered ( $\mathrm{pH} 7.40) 2.5 \%$ (vol/vol) glutaraldehyde fixative adjusted to an osmolality of 300 mosmol with sucrose (17).

Biochemical measurements. Sciatic nerve MI was determined gas liquid chromatographically in protein-free Somogyi filtrates of sciatic nerve homogenates as previously described $(11,20)$. Sciatic nerve $(\mathrm{Na}, \mathrm{K})$ ATPase activity was measured enzymatically as ouabain-inhibited ATPase activity in freshly prepared crude homogenates of whole sciatic nerve as previously described in detail (11). Briefly, nerve segments were minced and homogenized at $4^{\circ} \mathrm{C}$ in $2 \mathrm{ml}$ of $0.2 \mathrm{M}$ sucrose- $0.02 \mathrm{M}$ Tris$\mathrm{HCl} \mathrm{pH} \mathrm{7.5.} \mathrm{5-20} \mu$ l of homogenate was assayed spectrophotometrically in $1 \mathrm{ml}$ of $100 \mathrm{mM} \mathrm{NaCl}, 10 \mathrm{mM} \mathrm{KCl}, 2.5 \mathrm{mM} \mathrm{MgCl}, 1 \mathrm{mM}$ TrisATP, $1 \mathrm{mM}$ tri(cyclohexylammonium) phosphoenolpyruvate, $30 \mathrm{mM}$ imidazole- $\mathrm{HCl}$ buffer (pH 7.3), $0.15 \mathrm{mM}$ NADH, $50 \mu \mathrm{g}$ of lactate dehydrogenase and $30 \mu \mathrm{g}$ of pyruvate kinase (22). ATPase activity in the presence of $0.10 \mathrm{mM}$ ouabain was defined as the ouabain-uninhibitable ATPase fraction, since preliminary experiments had indicated that maximum ouabain inhibition is obtained at this concentration (11). Ouabaininhibitable ATPase activity, a precise measurement of ( $\mathrm{Na}, \mathrm{K})$-ATPase activity in rat sciatic nerve, was defined as the arithmetic difference between composite and ouabain-uninhibitable ATPase activities (11).

Teased fiber analysis. The proximal portion of the sural nerve was post-fixed and stained in cacodylate-buffered $1 \%$ (vol/vol) osmium tetroxide, and $54 \pm 3$ individual fibers were teased from each sural nerve segment in Epon (3). The teased fibers were examined under phase contrast illumination at a magnification of $\times 320$ and internodal length measured (3). The corresponding paranodal fiber diameter was determined by measuring the maximum fiber width within a $30-\mu \mathrm{m}$ distance of both nodes of Ranvier bordering each internodal segment, and the mean value recorded for internode (3). The mean internodal length for each fiber was plotted on the ordinate, and the paranodal fiber diameter was plotted on the abscissa, and the slope of the linear regression was used to define the paranodal fiber diameter to internodal length ratio (3).

Statistics. Data are mean \pm SEM, significance of difference is calculated by Student's $t$ test, and linear regressions are analyzed by the method of least squares.

\section{Results}

Clinical responses to insulin replacement, $A R I$, and MI therapy in $B B$ diabetic rats (Table I). At baseline, following $3 \mathrm{wk}$ of insulin deficiency, body weight was reduced, blood glucose was elevated, and tibial MNCV and EMPA were reduced similarly in all five diabetic groups compared with nondiabetic BB controls (Table I). Body weight, blood glucose, and tibial MNCV and EMPA remained abnormal for the additional 3-wk study period in insulin-deficient diabetics maintained on small doses of PZI (Table I). 3 wk of intensive insulin therapy in insulin replaced diabetics normalized the rate of weight gain (Table I) as well as blood glucose, MNCV and EMPA. Administration of either an ARI or MI for 3 wk to insulin-deficient BB rats did not affect body weight or blood glucose, but nearly completely normalized MNCV and EMPA in the ARI-treated and MI-treated diabetics.

Biochemical response in sciatic nerve to insulin replacement, $A R I$ and $M I$ therapy in insulin-deficient $B B$ rats (Table II). Sciatic nerve $M I$ content was significantly reduced in insulin-deficient diabetics compared with nondiabetic controls. As in the streptozotocin- $(20)$ and chronically diabetic $\mathrm{BB}(3,13)$ rat, intensive insulin therapy in the "insulin replaced" diabetics corrected the nerve MI depletion resulting from insulin deficiency (Table II). Both ARI and MI treatment also corrected nerve MI depletion in the ARI-treated and MI-treated BB diabetics as previously shown for the streptozotocin-induced diabetic rat (4-6, 20).

(Na,K)-ATPase activity. Neither 6 wk of sustained hyperglycemia in the insulin-deficient diabetics, nor 3 wk of unmodified insulin deficiency followed by 3 wk of ARI or MI administration statistically significantly altered composite ATPase activity in crude homogenates of sciatic nerve although composite activity tended to be lowest in the insulin-deficient diabetics (data not shown). However, the ouabain-inhibitable component of ATPase activity was reduced $\sim 28 \%$ in the insulin-deficient diabetics compared with the nondiabetic controls (Table II). 3 wk of intensive insulin therapy corrected this abnormality in the insulin- 
Table I. Effects of Insulin Replacement, ARI or MI Therapy on Metabolic Status and Tibial Nerve Conduction in Diabetic BB Rats

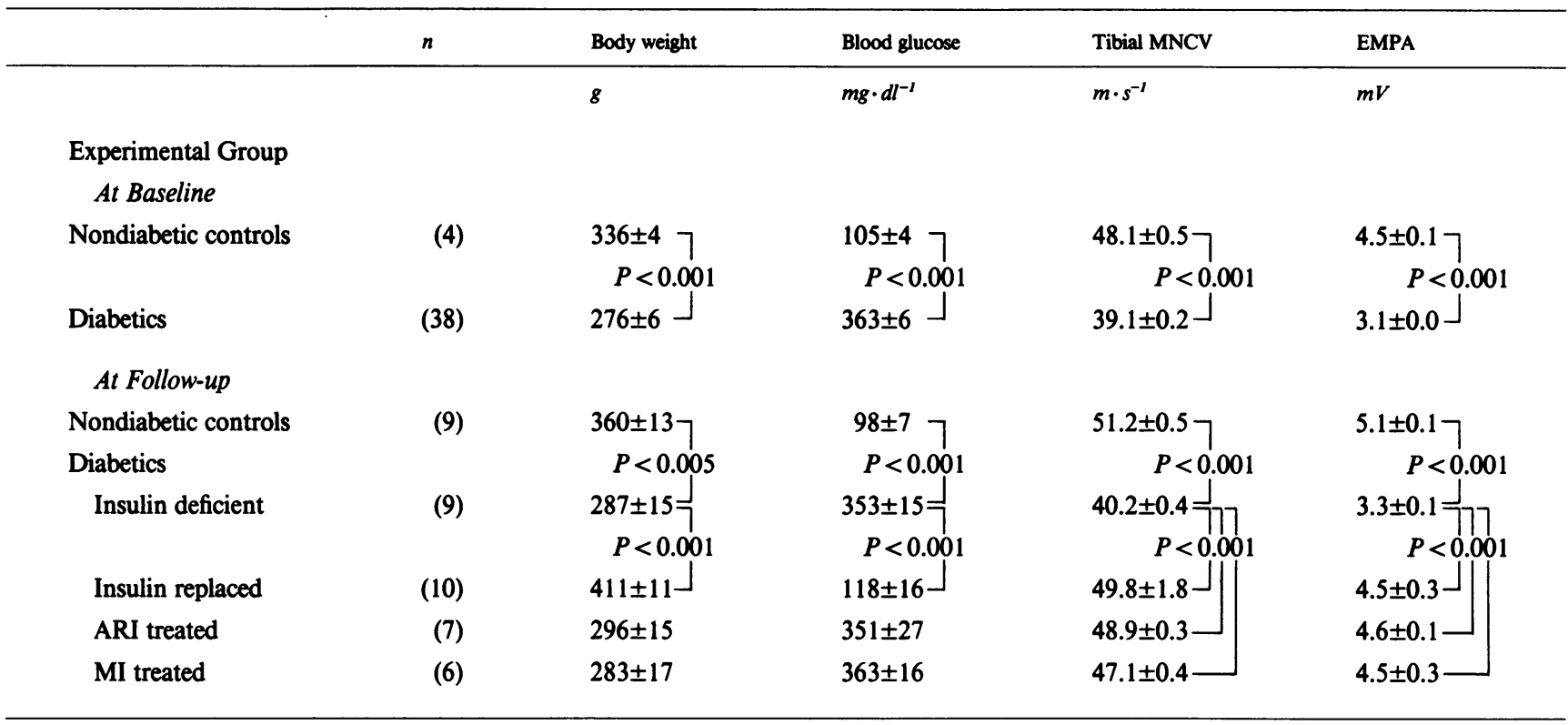

Diabetes-prone BB rats were monitored daily for spontaneous development of diabetes following which they were begun on small daily doses of insulin designed to assure survival but maintain hyperglycemia in the range of $350 \mathrm{mg} \cdot \mathrm{dl}^{-1}$. Age-matched non-diabetes-prone BB rats were used as nondiabetic controls. $3 \mathrm{wk}$ after the appearance of hyperglycemia i.e., at baseline, the diabetic rats were randomly assigned to the experimental groups. The baseline diabetics were immediately sacrificed for baseline morphometric studies, while the remaining groups were entered into a 3wk study period, during which the insulin-deficient diabetic rats were maintained hyperglycemic on small daily doses of insulin, the "insulinreplaced" diabetics were treated intensively with insulin to normalize blood glucose, and the "ARI-treated" and "MI-treated diabetics were maintained hyperglycemic on small doses of insulin along with either the ARI Statil $10 \mathrm{mg} \cdot \mathrm{kg}^{-1} \cdot \mathrm{d}^{-1}$ or $\mathrm{MI} 670 \mathrm{mg} \cdot \mathrm{kg}^{-1} \cdot \mathrm{d}^{-1}$ designed to normalize nerve MI content. Body wt, blood glucose, tibial MNCV and EMPA were recorded at the time of randomization of the diabetics into the five groups and again in the controls and the four remaining diabetic groups at the completion of the 3-wk study period. Values are shown as mean \pm SEM.

Table II. Effects of Insulin Replacement, ARI or MI Therapy on Sciatic Nerve MI Content and (Na,K)-ATPase Activity in Insulin-deficient BB Rats

\begin{tabular}{|c|c|c|c|c|}
\hline & \multirow[b]{3}{*}{$n$} & \multirow[b]{3}{*}{ MI content } & \multicolumn{2}{|l|}{ ATPase activity } \\
\hline & & & \multicolumn{2}{|l|}{ Ouabain- } \\
\hline & & & Inhibitable & Uninhibitable \\
\hline & & $\mathrm{mmol} \cdot \mathrm{kg}^{-1}$ & $\mu \mathrm{mol} \cdot \mathrm{g}^{-1} \cdot \mathrm{h}^{-1}$ & $\mu \mathrm{mol} \cdot \mathrm{g}^{-1} \cdot \mathrm{h}^{-1}$ \\
\hline \multicolumn{5}{|l|}{ Experimental group } \\
\hline Nondiabetics & (9) & $3.15+0.18 \neg$ & $121.8+5.77$ & $207+30$ \\
\hline Diabetics & & $P<0.010$ & $P<0.001$ & \\
\hline Insulin deficient & (9) & $\begin{array}{r}2.49+0.13777 \\
P<0.025\end{array}$ & $\begin{array}{r}83.8+4.2=77 \\
P<0.010\end{array}$ & $\begin{array}{r}176+14 \\
P<0.010\end{array}$ \\
\hline Insulin replaced & (8) & 3.18+0.18 ل & $120.0+7.4\rfloor \mid$ & $\begin{array}{r}259+23=7 \\
P<0.050\end{array}$ \\
\hline ARI treated & (7) & $3.16+0.21-$ & $116.9+4.4-$ & $175+28-$ \\
\hline MI treated & (6) & $3.48+0.32 \square$ & $113.3+8.3 \longrightarrow$ & $200+10$ \\
\hline
\end{tabular}

At the completion of the 3-wk study period, nondiabetic controls and BB diabetic rats treated as described in Methods and in Table I were anesthetized. Mid-thigh segments of the sciatic nerves were surgically removed, and either deproteinized and processed for gas-liquid chromatographic determination of MI, or homogenized in sucrose containing buffer for determination of composite and non-ouabain-inhibitable ATPase activity, using the enzymatic adenine-nucleotide-linked kinetic spectrophotometric method of Yoda and Yoda (22). Ouabain-inhibitable ATPase activity, a measure of $(\mathrm{Na}, \mathrm{K})$-ATPase activity in rat nerve $(7,11,13,17)$, was defined as the arithmetic difference between total and non-ouabain-inhibitable ATPase activity, and was expressed per mg wet wt of nerve. The differences between groups were not changed when the ATPase activities were expressed per mg Lowry protein (data not shown). Values for "insulin-replaced" diabetics are from a previously reported series (13) and are shown for comparison only. 
replaced diabetics. Aldose reductase inhibitor and MI therapy in the hyperglycemic BB rats increased sciatic nerve ouabaininhibitable ATPase activity in the ARI-treated and MI-treated diabetics to 94 and $97 \%$, respectively, of that in the nondiabetic control (Table II). In contrast, residual ATPase activity in the presence of inhibiting concentrations of ouabain ("ouabain uninhibitable ATPase activity") was similar across experimental groups (except the insulin-replaced diabetics whose composite activity was identical to their contemporaneous nondiabetic controls [13]). Thus, the effects of insulin deficiency and ARI or MI administration were primarily confined to the ouabaininhibitable $(\mathrm{Na}, \mathrm{K})$-ATPase fraction, which in rat sciatic nerve is a reliable estimate of $(\mathrm{Na}, \mathrm{K})$-ATPase activity $(7,11,13,17)$.

Qualitative assessment of teased fibers (Fig. 1). Teased myelinated nerve fibers from baseline and insulin-deficient diabetics exhibited marked swelling at or near the node of Ranvier compared with teased fibers from nondiabetic controls (3) (Fig. 1, compare panels $a$ and $b$ ). Paranodal swelling appeared as distinct cuffings around the paranodal area, or as swellings that progressively tapered off toward the internodal areas. This charac- teristic abnormality was encountered much less frequently in nerve fibers from insulin-replaced diabetics after $3 \mathrm{wk}$ of intensive insulin therapy, or from ARI-treated or MI-treated diabetics. Here the fibers had a much more normal appearance (Fig. $1 c$ and $d$ ).

Quantitative assessment of internodal length to paranodal fiber diameter ratio (Figs. 2 and 3). Linear regression analysis of internodal length vs. paranodal fiber diameter confirmed the impression that paranodal fiber diameter was enlarged in untreated insulin deficiency diabetes. Internodal fiber length and paranodal fiber diameter were linearly related in all six experimental groups (nondiabetic controls, and baseline, insulin-deficient, insulin-replaced, ARI-treated and MI-treated diabetics, Figs. 2 and 3). At baseline, the slope of the regression line was significantly shifted toward larger paranodal fiber diameters in the baseline diabetics compared with their age-matched nondiabetic controls $(P<0.001)$ (Fig. $2, a$ and $b)$.

At followup 3 wk later, the slope of the regression line was not significantly shifted in the older nondiabetic controls compared with the younger nondiabetic controls at baseline (compare
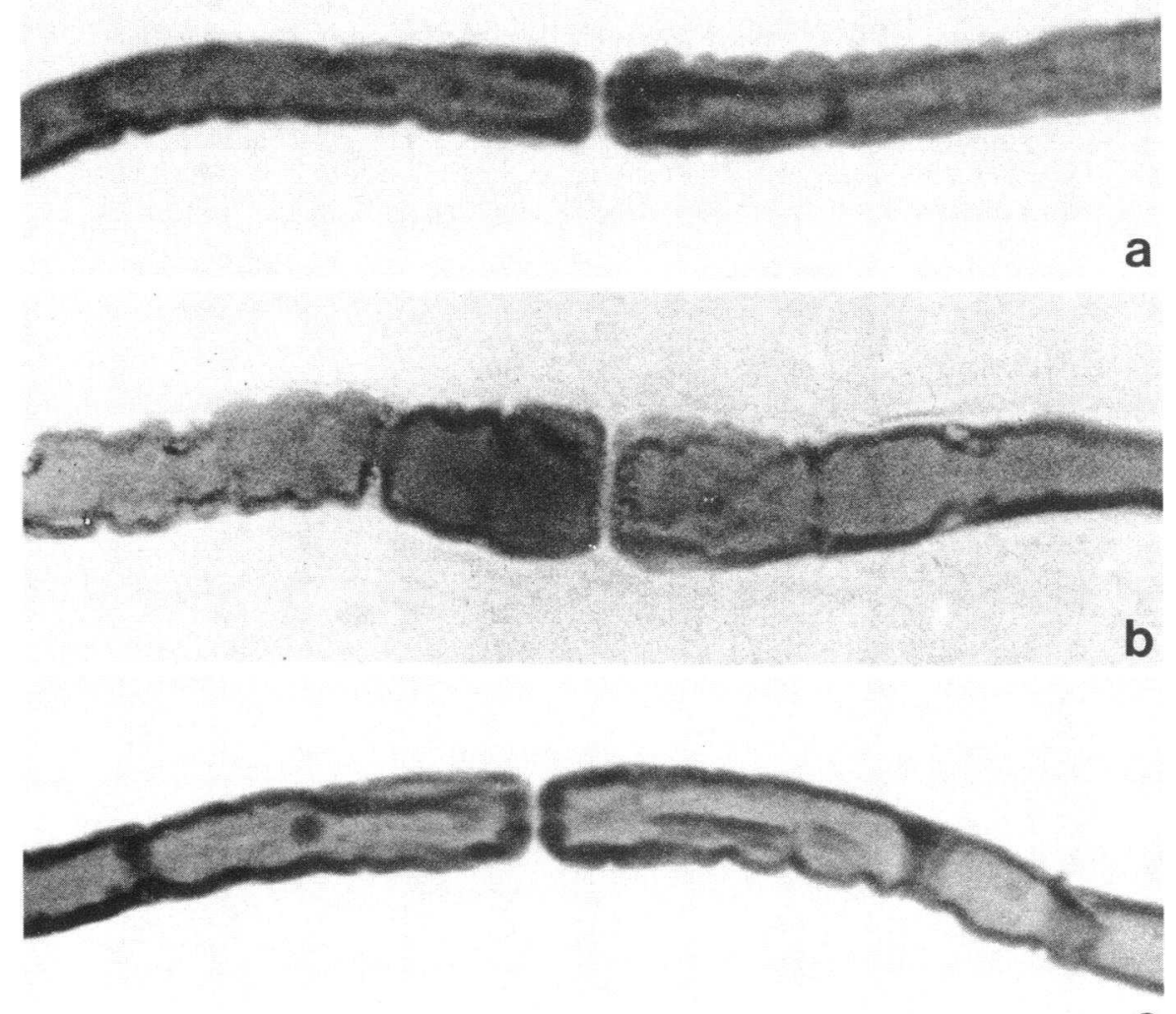

C

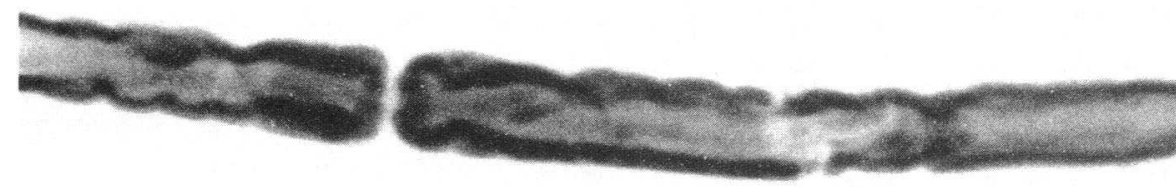

d
Figure 1. Effect of insulin deficiency, ARI treatment and MI supplementation on the appearance of the node of Ranvier of large myelinated fibers from the sural nerve of spontaneously diabetic $\mathrm{BB}$ rats. $\mathrm{BB}$ rats with spontaneous insulin deficiency of 3 wk duration were randomly distributed into experimental groups that remained insulin deficient without treatment, or were treated with an ARI or MI supplementation for an additional 3 wk. Photomicrographs of osmicated teased single fibers compare paranodal fiber appearance in $(a)$ a nondiabetic control rat, $(b)$ an insulin-deficient diabetic rat, $(c)$ an ARI-treated diabetic rat, and $(d)$ a MI-treated diabetic rat. Marked paranodal swelling that progressively tapered off toward the internodal areas was readily apparent in the untreated insulin-deficient diabetic rat $(b)$ compared to the nondiabetic control (a). Paranodal swelling was largely ameliorated after 3 wk of ARI- or MI-treatment ( $c$ and $d$ ), leaving fibers that resembled those of the nondiabetic control $(a)$. 


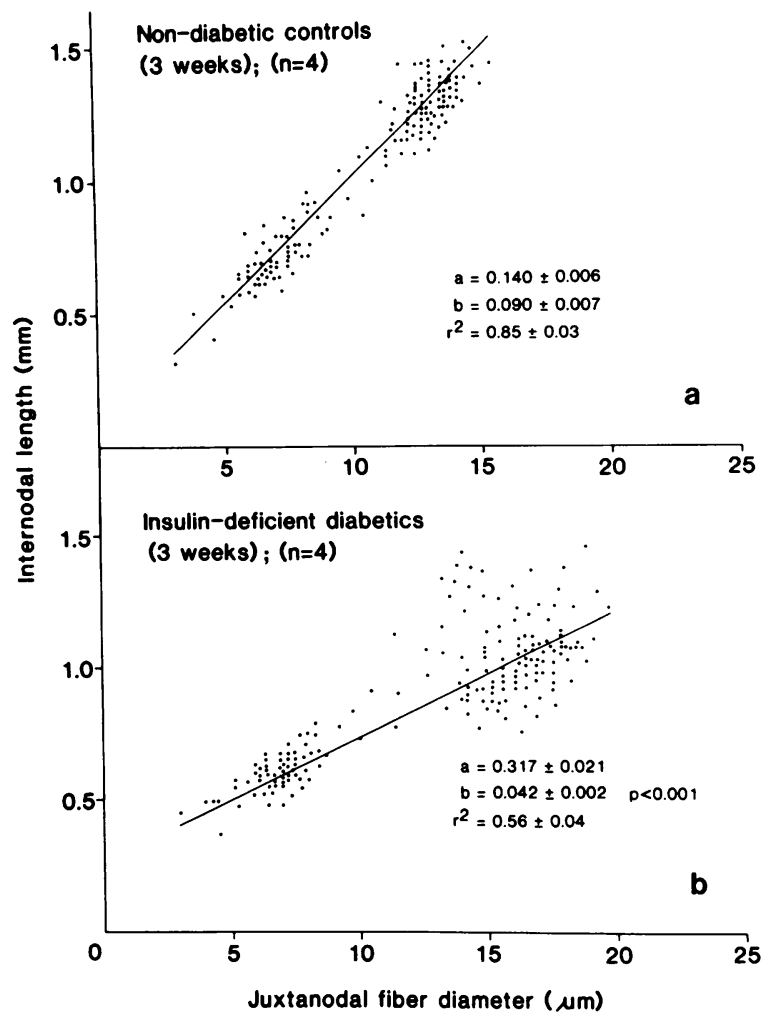

Figure 2. Linear regression analysis of internodal length vs. paranodal fiber diameter in nondiabetic controls $(a)$ and baseline diabetics $(b)$ at the beginning of the 3-wk study period. The analysis showed a signifcant shift of the slope of the regression line toward larger paranodal fiber diameters in the baseline diabetics $(b)$ confirming the impression, illustrated in Fig. 1, that insulin deficiency is assaciated with paranodal swelling.

Figs. 2 and $3 a$ ). The slope of the regression line was significantly shifted toward larger fiber diameters in insulin-deficient diabetics compared with their age-matched nondiabetic controls at followup $(P<0.001)$ (Fig. 3, $a$ and $b$ ). Intensive insulin therapy, or administration of an ARI or MI significantly increased the slope of the regression lines in the insulin-replaced, ARI-treated and MI-treated diabetics (Fig. 3, $c-e$ ) (all $P<0.001$ vs. both baseline and insulin-deficient diabetics) rendering them statistically indistinguishable from that of the baseline and followup nondiabetic controls. These alterations in the ratio of internodal length to paranodal fiber diameter could not be explained on the basis of shortened internodes in the baseline or insulin-deficient diabetics, since the ratio of internodal length to internodal fiber diameter was the same, and the mean internodal lengths were unaltered among the six experimental groups (data not shown). These data are consistent with the conclusion that paranodal fiber diameter was increased in the baseline and insulin-deficient diabetics, and that insulin replacement therapy or administration of either an ARI or MI completely reversed this phenomenon.

\section{Discussion}

The widely held view that the metabolic factors responsible for acutely reversible functional impairment in diabetic peripheral nerve also contribute to the characteristic structural alterations of diabetic neuropathy has never been satisfactorily established on an experimental basis (2). Hyperglycemia in man (23) and the rat $(5,6,20)$ induces two metabolic defects in peripheral nerve strongly associated with reversible slowing of nerve conduction: sorbitol accumulation and MI depletion (2). Theoretically, these two glucose-related metabolic abnormalities could be linked to paranodal swelling on an osmotic basis, either by the intracellular accumulation of osmotically active sorbitol (18), or by the MI-related (Na,K)-ATPase defect (1-3). The two relevant enzymes, aldose reductase (24) and ( $\mathrm{Na}, \mathrm{K})$-ATPase (2527), are concentrated in the paranodal region of large myelinated nerve fibers, the former primarily in the paranodal Schwann cell cytoplasm (24), and the latter both there and in the paranodal axolemma (25). Hence alterations in the activities of these two enzymes might understandably induce swelling confined to the paranodal region.

Insulin treatment reverses MI depletion, (Na,K)-ATPase deficiency (13) and sorbitol accumulation (28) in diabetic rats. Administration of an ARI or MI supplementation, both of which prevent or reverse $\mathrm{MI}$ depletion and $(\mathrm{Na}, \mathrm{K})$-ATPase deficiency in diabetic peripheral nerve $(4-7,11)$, reverses paranodal swelling despite persistent insulin deficiency and hyperglycemia. Because ARIs but not MI supplementation decrease nerve sorbitol levels (3), MI depletion rather than sorbitol accumulation appears to be the necessary element for sustained paranodal fiber swelling in the insulin deficient BB rat between 3 and 6 wk of diabetes. Presumably paranodal swelling would be prevented by therapy that blocks the initial depletion of $\mathrm{MI}$ and reduction in $(\mathrm{Na}, \mathrm{K})$ ATPase activity $(4-7,11)$, and the resultant rise in intracellular sodium shortly after the onset of diabetes (3). Ultrastructural and electrophysiological studies equating paranodal swelling with sodium accumulation $(3,15)$ and distention $(3,19)$ in the axon rather than the Schwann cell, combined with the identification of $(\mathrm{Na}, \mathrm{K})$-ATPase $(25)$ but not aldose reductase $(24,29)$ in the axon, further implicate the MI-related (Na,K)-ATPase defect rather than sorbitol accumulation in the swelling of large myelinated nerve fibers in the BB rat. On the other hand, the largely unanticipated but often confirmed observation that inhibitors of the enzyme aldose reductase, located primarily in the Schwann cell, prevent the axonal $(1,3,15)$ MI-related (Na,K)-ATPase defect $(4-7,11)$, implies the existence of unexplored but physiologically important interactions between the axon and Schwann cells at the node of Ranvier.

Paranodal swelling abates with the appearance of axo-glial dysjunction in the BB rat $(3,17)$, presumably reflecting the diminished demand on (Na,K)-ATPase-dependent sodium extrusion following nerve depolarization conferred by the associated decrease in nodal voltage-dependent sodium permeability (3, 15). Paranodal swelling and axo-glial dysjunction correlate closely in sural nerve biopsies from patients with chronic neuropathy complicating insulin-dependent diabetes mellitus (IDDM), and are both related to duration and severity of hyperglycemia (19). Mechanical stresses associated with paranodal swelling presumably contribute to the disruption of junctional complexes between the axolemma and terminal loops of myelin, resulting in axo-glial dysjunction (17). Hence, for the first time, a defined cause and effect chain of metabolic, biophysical, and ultrastructural abnormalities has emerged linking insulin deficiency and hyperglycemia, on one hand, with characteristic structural abnormalities in human and murine diabetic neuropathy on the other, i.e., paranodal swelling and axo-glial dysjunction. These structural lesions account for poorly reversible nerve conduction slowing in diabetic rats $(13,17)$, and are viewed 


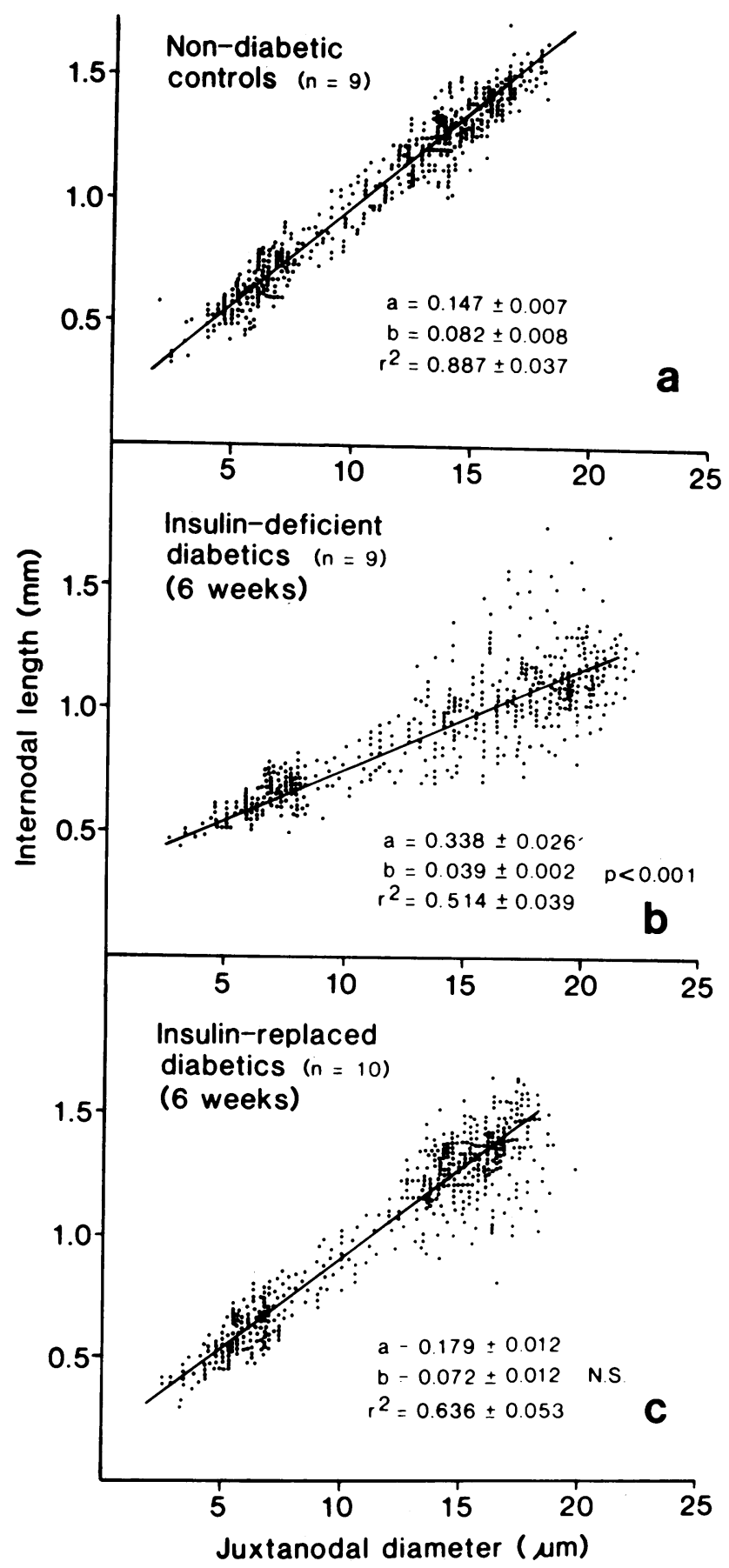

Figure 3. Linear regression analysis of internodal length vs. paranodal fiber diameter in the nondiabetic controls and 4 diabetic experimental groups at the conclusion of the $3 \mathrm{wk}$ study period. (a) Nondiabetic controls, $(b)$ insulin-deficient diabetics, $(c)$ insulin-replaced diabetics, $(d)$ ARI-treated diabetics, and (e) MI-treated diabetics. $6 \mathrm{wk}$ of insulin deficiency significantly shifted the slope of the regression line towards

as a precursor to paranodal demyelination and probably nerve fiber loss in patients with diabetic neuropathy (19). Intervening links in the chain are the glucose-mediated defects in polyol and MI metabolism, phosphoinositide turnover, diacylglycerol release, protein kinase $\mathrm{C}$ activation and $(\mathrm{Na}, \mathrm{K})-\mathrm{ATPase}$ function that cause intracellular sodium accumulation and resultant ax-

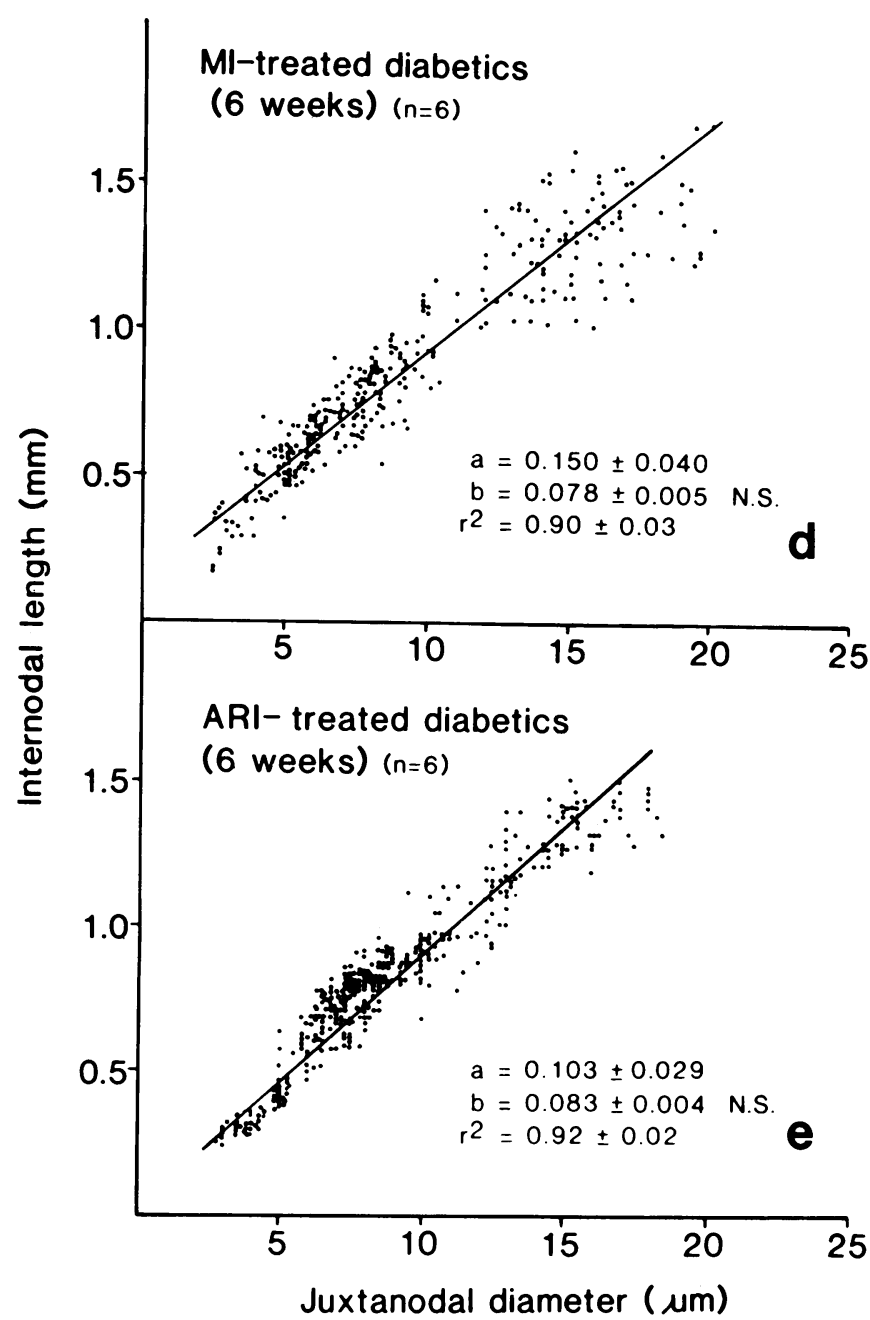

larger paranodal fiber diameters in the insulin-deficient diabetics $(b)$ compared to the age-matched nondiabetic controls $(a)$, confirming the presence of paranodal swelling. $3 \mathrm{wk}$ of insulin replacement (c), ARItreatment $(d)$, or MI supplementation $(e)$ reversed this paranodal swelling, rendering the slope of the linear regression statistically indistinguishable from the nondiabetic control $(a)$.

onal swelling (1-3). The studies reported in this communication thus significantly clarify and strengthen the argument that the metabolic abnormalities previously implicated in acute functional impairment in diabetic peripheral nerve also play an important role in the development of chronic structural alterations characterizing diabetic polyneuropathy. 


\section{Acknowledgments}

These studies were supported in part by U. S. Public Health Service grant RO-1AM-29892, the Harry Soffer Memorial Research Fund of the University of Pittsburgh, the Canadian Diabetes Association, the Medical Research Council of Canada grant MA-7777, and a Research Grant from Stuart Pharmaceuticals, Wilmington, DE.

\section{References}

1. Sima, A. A. F. 1985. Annotation. Can the BB-rat help to unravel diabetic neuropathy? Neuropathol. Appl. Neurobiol. 11:253-264.

2. Greene, D. A., S. Lattimer, J. Ulbrecht, and P. Carroll. 1985. Glucose-induced alterations in nerve metabolism: current perspective on the pathogenesis of diabetic neuropathy and future directions for research and therapy. Diabetes Care. 8:290-299.

3. Sima, A. A. F., and T. Brismar. 1985. Reversible diabetic nerve dysfunction: structural correlates to electrophysiological abnormalities. Ann. Neurol. 18:21-29.

4. Finegold, D., S. Lattimer, S. Nolle, M. Bernstein, and D. A. Greene. 1983. Polyol pathway activity and myo-inositol metabolism. Diabetes. 32:988-992.

5. Gillon, K. R. W., and J. N. Hawthorne. 1983. Sorbitol, inositol and nerve conduction in diabetes. Life Sci. 32:1943-1947.

6. Mayer, J. H., and D. R. Tomlinson. 1983. Prevention of defects of axonal transport and nerve conduction velocity by oral administration of myo-inositol or an aldose reductase inhibitor in streptozotocin-diabetic rats. Diabetologia. 25:433-438.

7. Greene, D. A., and S. A. Lattimer. 1984. Action of sorbinil in diabetic peripheral nerve. Relationship of polyol (sorbitol) pathway inhibition to a myo-inositol-mediated defect in sodium-potassium ATPase activity. Diabetes. 33:712-716.

8. Greene, D. A., and S. A. Lattimer. 1982. Sodium- and energydependent uptake of myo-inositol by rabbit peripheral nerve: competitive inhibition by glucose and lack of an insulin effect. J. Clin. Invest. 70: 1009-1018.

9. Simmons, D. A., A. I. Winegrad, and D. B. Martin. 1982. Significance of tissue myo-inositol concentrations in metabolic regulation in nerve. Science (Wash. DC). 217:848-851.

10. Das, P. K., G. Bray, J. A. Aquayo, and M. Rasminsky. 1976. Diminished ouabain-sensitive, sodium-potassium ATPase activity in sciatic nerves of rats with streptozotocin-induced diabetes. Exp. Neurol. 53:285-288.

11. Greene, D. A., and S. A. Lattimer. 1983. Impaired rat sciatic nerve sodium-potassium adenosine triphosphatase in acute streptozocin diabetes and its correction by dietary myo-inositol supplementation. $J$. Clin. Invest. 72:1058-1063.

12. Greene, D. A., and S. A. Lattimer. 1984. Impaired energy utilization and (Na,K)-ATPase in diabetic peripheral nerve. Am. J. Physiol. 246:E311-E318.

13. Greene, D. A., S. Yagihashi, S. A. Lattimer, and A. A. F. Sima. 1984. Nerve $\mathrm{Na}^{+}-\mathrm{K}^{+}-\mathrm{ATPase}$, conduction and myo-inositol in the insulin deficient BB rat. Am. J. Physiol. 247:E534-E539.
14. Greene, D. A., and S. A. Lattimer. 1986. Protein kinase C agonists acutely normalize decreased ouabain-inhibitable respiration in diabetic rabbit nerve: implications for $(\mathrm{Na}, \mathrm{K})-\mathrm{ATPase}$ regulation and diabetic complications. Diabetes. 35:242-245.

15. Brismar, T., and A. A. F. Sima. 1981. Changes in nodal function in nerve fibers of the spontaneously diabetic BB-Wistar rat. Potential clamp analysis. Acta Physiol. Scand. 113:499-506.

16. Brismar, T. 1983. Nodal function of pathological fibers. Experientia (Basel). 39:946-953.

17. Sima, A. A. F., S. A. Lattimer, S. Yagihashi, and D. A. Greene. 1986. Axo-glial dysjunction. A novel structural lesion that accounts for poorly-reversible conduction slowing in the spontaneously-diabetic Biobreeding rat. J. Clin. Invest. 77:474-485.

18. Gabbay, K. H. 1973. The sorbitol pathway and the complications of diabetes. N. Engl. J. Med. 288:831-836.

19. Sima, A. A. F., V. Bril, and D. A. Greene. 1986. A new characteristic ultrastructural abnormality, and morphologic evidence for pathogenetic heterogeneity in human diabetic neuropathy. Clin. Res. 34:688a. (Abstr.)

20. Greene, D. A., P. V. DeJesus, and A. I. Winegrad. 1975. Effects of insulin and dietary myoinositol on impaired peripheral motor nerve conduction velocity in acute streptozotocin diabetes. J. Clin. Invest. 55: 1326-1336.

21. Sima, A. A. F., and K. Hay. 1981. Functional aspects and pathogenetic considerations of the neuropathy in the spontaneously diabetic BB-Wistar rat. Neuropathol. Appl. Neurobiol. 7:341-350.

22. Yoda, A., and S. Yoda. 1980. A new simple method for Na,KATPase rich membrane fragments. Anal. Biochem. 110:82-88.

23. Mayhew, J. A., K. R. W. Gillon, and J. N. Hawthorne. 1983. Free and lipid inositol, sorbitol and sugars in sciatic nerve obtained postmortem from diabetic patients and control subjects. Diabetologia. 24: 13-15.

24. Chakrabarti, S., A. A. F. Sima, and S. Yagihashi. 1986. Aldose reductase in the BB rat; purification, immunological identification and localization in the retina and peripheral nerve. Diabetes. 35:101A.

25. Vorbrodt, A. W., A. S. Lossinsky, and H. M. Wisniewski. 1982. Cytochemical localization of ouabain-sensitive $\mathrm{K}^{+}$-dependent, p-nitrophenylphosphatase (transport ATPase) in the mouse central and peripheral nervous systems. Brain Res. 243:225-234.

26. Wood, J. G., D. H. Jean, J. N. Whitaker, B. J. McLaughlin, and R. W. Albers. 1977. Immunocytochemical localization of sodium, potassium activated ATPase in knifefish brain. J. Neurocytol. 6:571-581.

27. Schwartz, M., S. A. Ernst, G. J. Siegel, and B. W. Agranoff. 1981. Immunocytochemical localization of $\left(\mathrm{Na}^{+}, \mathrm{K}^{+}\right)$-ATPase in the goldfish optic nerve. J. Neurochem. 36:107-115.

28. Stewart, M. A., W. R. Sherman, M. M. Kurien, G. I. Moonsammy, and M. Wisgerhof. 1967. Polyol accumulations in nervous tissue of rats with experimental diabetes and galactosaemia. J. Neurochem. 14:10571066.

29. Ludvigson, M. A., and R. L. Sorenson. 1980. Immunohistochemical localization of aldose reductase. I. Enzyme purification and antibody preparation-localization in peripheral nerve, artery, and testis. Diabetes. 29:438-449. 